\title{
Timing Properties and Correctness for Structured Parallel Programs on $x 86-64$ Multicores
}

\author{
Kevin Hammond, Christopher Brown, and Susmit Sarkar \\ School of Computer Science, University of St Andrews, Scotland, UK. \\ \{kevin.hammond, susmit.sarkar, cmb21\}@st-andrews.ac.uk
}

\begin{abstract}
This paper determines correctness and timing properties for structured parallel programs on x86-64 multicores. Multicore architectures are increasingly common, but real architectures have unpredictable timing properties, and commonly used relaxed-memory concurrency models mean that even functional correctness is not obvious. This paper takes a rigorous approach to correctness and timing properties, examining common locking protocols from first principles, and extending this through queues to structured parallel constructs. We prove functional correctness and derive simple timing models, extending these for the first time from low-level machine operations to high-level parallel patterns. Our derived high-level timing models for structured parallel programs allow us to accurately predict upper bounds on program execution times on x86-64 multicores.
\end{abstract}

Keywords: Multicore, relaxed-memory concurrency, functional correctness, algorithmic skeletons, operational semantics, timing models.

\section{Introduction}

Multicore architectures are increasingly common, providing excellent trade-offs between performance and energy consumption. However, the actual execution behaviour of parallel programs on real multicores is still often difficult to understand. Arguing about the correctness of parallel programs is non-trivial in the presence of real-world relaxed-memory consistency models. Moreover, predicting the execution time of parallel programs is hard. Both issues derive from the same problem: there is no well-understood correspondence between the high-level parallel primitives that programmers use and the low-level implementations of those primitives that are actually executed. We address this problem by directly considering correctness and timing properties from basic machine-level operations all the way to high-level parallelism structures.

This paper exploits structured parallel programming, in the form of algorithmic skeletons [1,2]. Such approaches bring similar abstraction advantages to parallel program design that standard structured programming brings to sequential programming, abstracting over basic parallelism primitives, such as process creation, communication and synchronisation. 


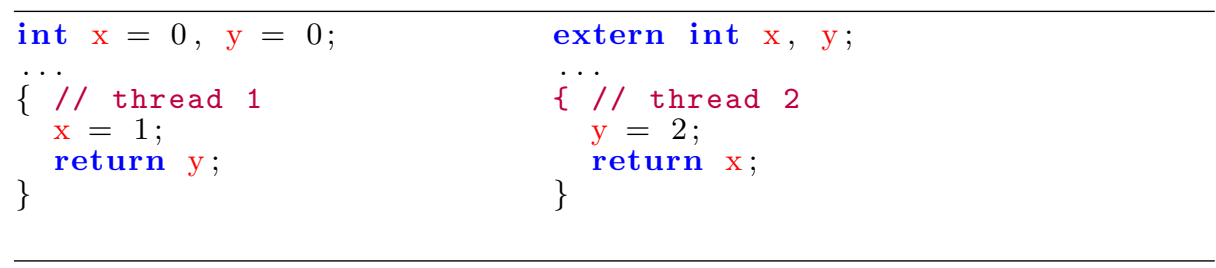

Fig. 1. Under the $x 86$ - TSO relaxed-memory consistency model, both threads could return 0 . This is not possible under SC.

The main advantages to us are:

1. The programmer can think in terms of patterns of parallelism, rather than e.g. low-level memory operations;

2. Certain complex parallel conditions, such as race conditions and deadlocks, are eliminated by design, so dramatically simplifying design, implementation and testing of parallel programs; and

3. Simple, but effective, performance models are possible.

This paper exploits these advantages to produce strong and principled cost models for two fundamental parallel programming structures: task farms and parallel pipelines. Danelutto et al. [3] have shown that many common parallelism patterns can be expressed in terms of these two primitives. Our work thus extends straightforwardly to many other high-level patterns of parallelism.

Memory Consistency. Standard correctness proofs of parallel programs usually assume memory accesses to be interleaved, so-called Sequential Consistency (SC) [4]. However, x86-64 multicores, and many other recent architectures (e.g. ARM and IBM Power), do not comply with this assumption, making such reasoning invalid. Consider, for example, the program fragment in Figure 1. Under the Total Store Order relaxed-memory consistency model that is used by $x 86-64$ multicores $(x 86$-TSO), both threads could return 0 , a result that is impossible using the simpler SC model.

Novel Contributions. While there has been significant previous work on algorithmic skeletons, this paper represents the first attempt, of which we are aware, to establish cost models from first principles for widely-used multicore hardware. In addition, this paper makes the following specific novel contributions:

1. It describes an operational semantics for structured parallel programs, that is used to derive, from first principles, a compositional cost model for any combination of the farm and pipeline algorithmic skeletons;

2. It gives a simple operational proof of the (partial) correctness of a widelyused spin-lock protocol using the actual relaxed-memory concurrency semantics used by $x 86-64$ multicore machines; the proof composes in a straightforward fashion to show that the farm and pipeline algorithmic skeletons are 


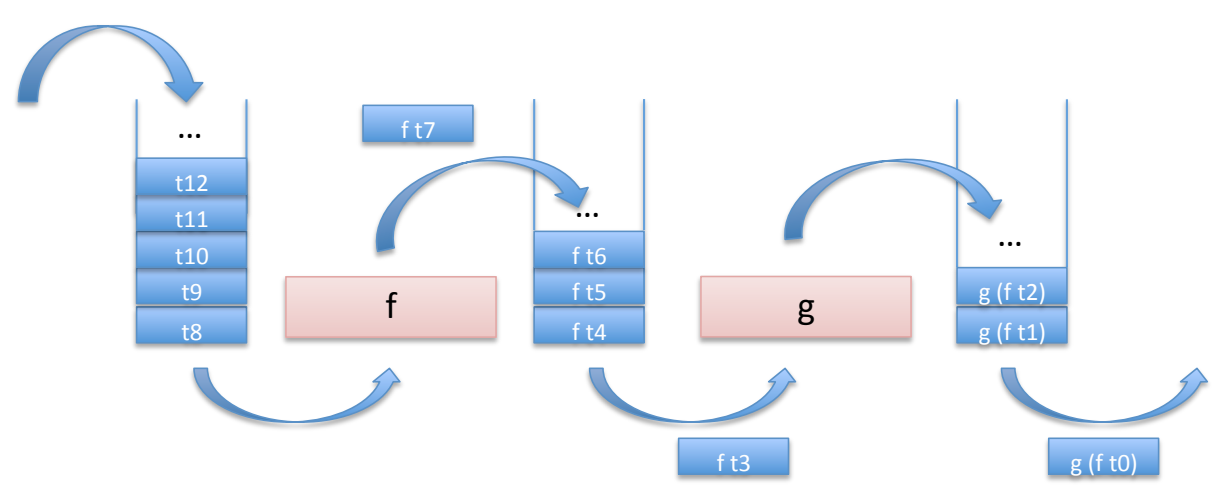

Fig. 2. Two-Stage Parallel Pipeline: $f \mid g$

free from deadlock, both singly and in any combination, when implemented by queues using that locking protocol; and

3. It validates the cost model against some example parallel programs, giving accurate predictions of lower-bound speedups; our predictions are typically within $7 \%$ and in all cases within $30 \%$ of the actual speedup.

A key aspect of our approach is that we model the actual relaxed-memory model used by real $x 86-64$ multicore systems, exploiting recent work on the $x 86$-TSO model [5,6], which gives a precise account of the observable behaviour of $x 86-64$ multiprocessors in terms of an idealised abstract machine. We are thus able, for the first time, to provide realistic cost models for programs that are executed in parallel on such architectures. The spin-lock protocol that we use has been shown to be the most efficient protocol for implementing simple locks on $x 86$ - 64 architectures [5]. It is widely used to implement real parallel programs. Our correctness proof is the first direct operational proof that this protocol is correctly implemented by the underlying hardware through its relaxed-memory access protocol. Finally, the queue protocol shown here has many uses beyond algorithmic skeletons, and is representative of many widely-used higher-level synchronisation protocols. The correctness proof shown here is likewise the first proof that such a protocol is correctly implemented by the underlying multicore hardware.

\section{Pipeline and Farm Skeletons}

In this paper, we consider two fundamental skeletons: 2-stage pipelines (Figure 2) and task farms (Figure 3). We use a streaming implementation that links skeletons using (unbounded) queues. This allows skeletons to be easily composed, and also allows arbitrarily nested parallel structures to be built from the basic skeletons. Parallel pipelines $(f \mid g)$ represent the composition of two operations $f$ and $g$, streamed over a sequence of inputs $t_{0}, t_{1}, \ldots$, with $f$ and $g$ possibly executed in parallel. The result of the pipeline is the stream $g\left(f t_{0}\right), g\left(f t_{1}\right), \ldots$ 


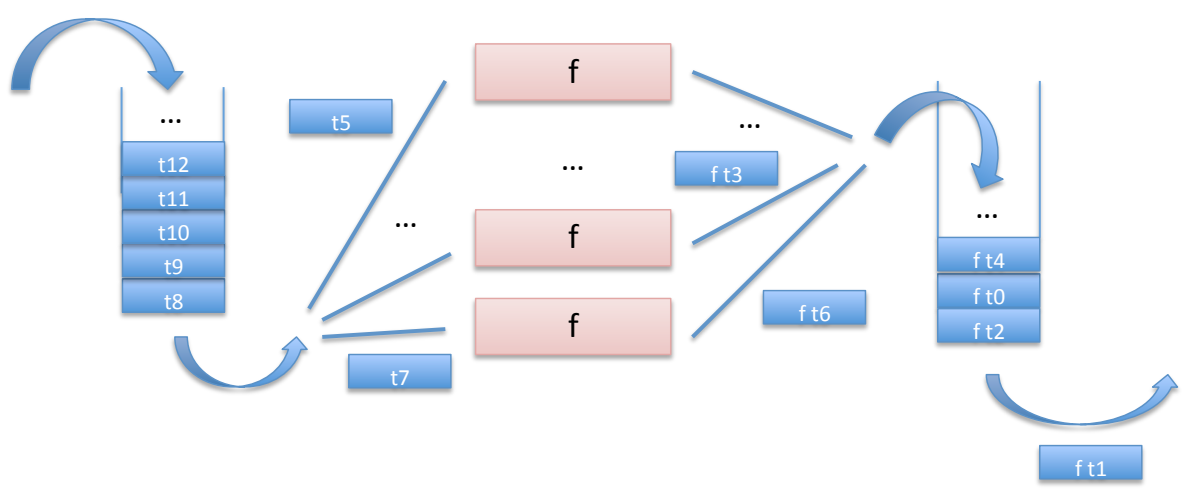

Fig. 3. Task Farm: $\Phi(f)$

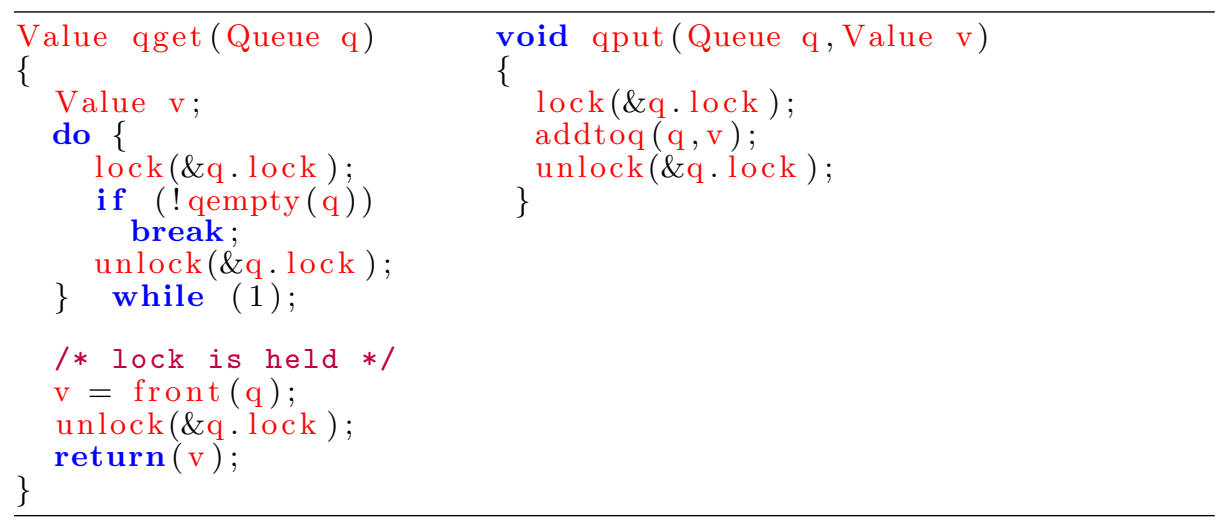

Fig. 4. Simple queue implementation in C.

Multi-stage pipelines can be built by composing multiple two-stage pipelines and then merging the input/output queues. Task farms $(\Phi(f))$ apply the same operation $f$ to each of the inputs in a stream. A fixed number of worker instances is created, which each apply $f$ in parallel to a subset of the inputs. The result of applying a farm to a stream of inputs $t_{0}, t_{1}, \ldots$ is then the bag $\left\{f t_{0}, f t_{1}, \ldots\right\}$, where the results may be produced in an arbitrary order. This non-deterministic definition of a task farm allows an efficient parallel implementation, where each of the workers is mapped to a different processing agent. As each worker produces its result, it is placed in the output queue, and the worker takes the next input (if any) from the input queue. Farms can be nested to an arbitrary depth by replacing the operation $f$ by a farm and linking the corresponding input and output queues. It is also possible to embed pipelines within farms or vice-versa, so yielding arbitrarily complex parallel systems. 


\subsection{Simple Queue Implementation using Locks}

The pipeline and farm skeletons use queues to manage the input/output streams. Figure 4 shows how queues can be implemented using lock and unlock primitives, plus operations to remove the first element from the queue (front), add a new element to the queue (addtoq), and check whether the queue is empty (qempty). Queues are implemented using the abstract type Queue, containing values of type Value. The qget operation returns the first element from the queue, spinning if the queue is empty. This implements a blocking read operation. The corresponding qput operation adds a value to the end of the queue, locking and unlocking as necessary. When used as a pair, qget/qput implement a synchronisation operation between two parallel threads. Figure 5 gives a spinlock implementation of lock/unlock in $\mathrm{C}$ and x86-64 assembler, assuming an atomic exchange primitive, exchange. Spin-locks are widely used in parallel systems where there are low contention rates, since they are simple to implement and the costs of acquiring and releasing the locks are very low. In fact, until recently, the Linux kernel used almost identical code. For fairness reasons, it now uses a slightly modified version (ticketed spin-locks). Here, lockcell is a variable that contains the lock. If it has the value 1 , then the lock has been acquired by some thread; if it has the value 0 , then no thread has acquired the lock. Acquiring the lock using the lock routine involves reading the value of lockcell, and exchanging it with the locked value, 1 . If the lock has already been acquired by another thread, the process is repeated until the lock can be acquired (i.e. the previous value of lockcell was 0). Releasing the lock using the unlock routine simply involves setting lockcell to 0 . Note that the unlock need not be an atomic memory operation.

\section{Key Hardware Characteristics}

In what follows, we will consider memory accesses to be classified into Reads from and Writes to specific memory locations, plus Fences and atomic Exchanges. Intel's x86-64 instruction set also provides some refined versions of these basic operations [7]. Sequential consistency (SC) memory models [4] ensure that memory accesses from multiple threads are carried out in an order that is consistent with some valid set of memory accesses by a fully sequential processor. That is, memory accesses from different threads are interleaved so that there is effectively a single thread of memory accesses. Recall the simple example from Figure 1. Here, depending on the exact timing of Reads and Writes on $x$ and $y$, thread 1 could return either 1 or 0 and thread 2 could return either 2 or 0 . It is not possible for both threads to return 0, however, since one of the two Writes to $x$ or $y$ must happen last. While $\mathrm{SC}$ is effective on uni-processor systems, enforcing an SC memory model on a multicore system can carry significant performance penalties. For example, all caches and other memory hardware must be synchronised in order to avoid inconsistent results. Since such a strong model is not always required, multicore hardware vendors generally support weaker 


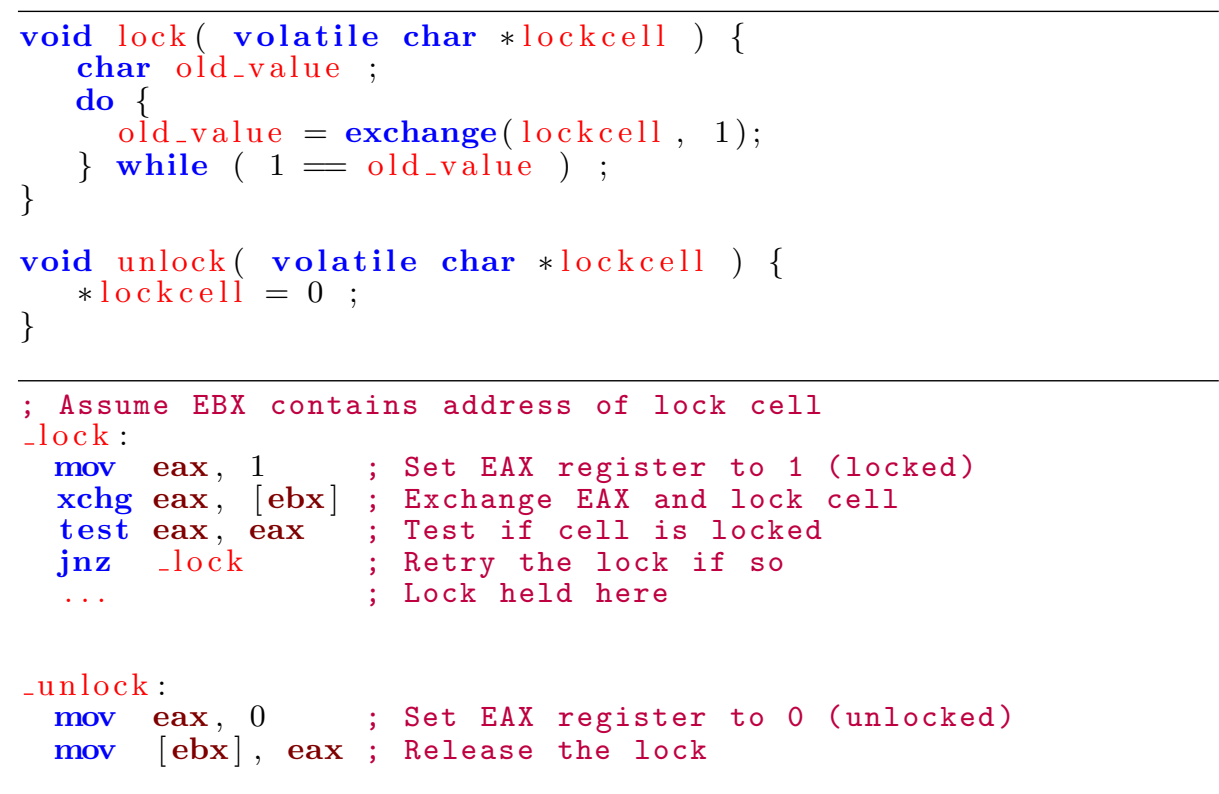

Fig. 5. Above, simple spin-lock implementation in pseudo-C using an atomic exchange operation on the lockcell memory location; 1 indicates the lock is acquired; 0 is used to release the lock. Below, corresponding x86-64 instructions (ignoring function prelude/postlude). The xchg instruction in llock acquires the lock, which is released in in _unlock using a normal memory write. Note that on x86-64, a xchg implicitly behaves as a full memory barrier.

consistency models that offer higher performance. Modern $x 86$-64-class microprocessors use Total Store Ordering (x86-TSO) [6,5]. $x 86$-TSO guarantees that the order in which Write instructions for a given processor appear in (shared) memory is identical to the sequence in which the processor issued the Writes. $x 86$ - TSO can be implemented by providing each hardware thread of execution with a private FIFO write-buffer. This is an abstract machine implementation: a more realistic hardware implementation is discussed further below. Writes are stored temporarily in this buffer prior to being actioned by the main memory. Reads from the local processor (only) can access this write buffer, if necessary, as an intermediate step between reading from the private and shared caches. Writes recorded in the write buffer will be used in preference to any corresponding values in the shared cache. After an unpredictable, but finite, time, each Write is flushed from the buffer, and so becomes visible to all processors. In this way, SC is enforced for a single processor, but not for all processors in a multi-processor system. In the $x 86$-TSO model, explicit memory fence (mfence) or atomic exchange (xchg) instructions are needed to enforce consistency across multiple processors. When a Fence/Exchange instruction is encountered by a processor, all of its outstanding Reads and Writes are executed immediately. 
This provides strong local temporal guarantees: all local accesses that appeared in the instruction stream prior to a Fence/Exchange will be executed before any accesses that appeared after the Fence/Exchange. It also provides strong global memory guarantees: all memory locations (and any cached copies) will be consistent with the memory state immediately following the Fence/Exchange.

Definition 1. Thread. A Thread is an ordered sequence of memory accesses, where memory accesses comprise Reads, Writes, Fences and Exchanges to specific memory locations. A Read takes a memory location and returns a value. A Write takes a memory location and a value and has no result. A Fence has no parameters or result. An Exchange is treated as a simultaneous and indivisible Read, Write and Fence. It takes a memory location and a value and returns a (possibly different) value.

Definition 2. Write buffer. A Write buffer is associated with each thread, and contains an ordered sequence of Writes, each associating a value with an address. Each Write is recorded in the corresponding write buffer immediately it is executed by any Thread.

Definition 3. Memory. A Memory maps addresses to values.

Definition 4. Core. A Core comprises a set of Threads.

Definition 5. Multicore. A Multicore comprises a set of Cores plus a single shared Memory ${ }^{1}$.

Definition 6. Execution Order. The execution order is a linear trace of transitions made by the labelled transition system called the x86-TSO machine [6].

Hardware Correspondence The abstract model above talks about "threads" of execution. In current multiprocessor implementations, these are grouped together in various ways, each of which has varying implications for scheduling and timing. Firstly, user-level threads are mapped by the runtime system to hardware-level threads. In this paper, we ignore this scheduling cost. That is, we consider only a non-preemptive model with no explicit thread yields to other threads or the runtime system. Taking such yields into account would introduce interference with other processes and threads running on the system. The model above speaks of such hardware threads when mentioning a thread of execution. Secondly, each hardware thread is mapped to specific hardware resources on a core. The mapping may be one-to-one, or many-to-one (hyperthreading, also known as simultaneous multi-threading). Each hyperthread generally has exclusive use of registers and a load-store reordering buffer. The load-store reordering buffer maintains metadata to ensure observable FIFO buffering for the stores, and also to ensure that loads appear ordered to the programmer, even though

${ }^{1}$ We will ignore shared cache here, since it does not have a significant impact on the proofs. 
aggressive implementations can and do perform out-of-order operations, e.g. satisfaction of read requests. Third, a set of cores (typically 2 to 4 ) are collected in one CPU. Typically, the first levels of cache (L1 and L2 on the Intel Core-i7) are private to the core, while higher levels (L3 on the Intel Core-i7) are shared between all the cores on one CPU. Finally, multiple CPUs may be connected in one system, all sharing a common memory. The caches communicate with each other and with memory to maintain "cache coherence", that is, a clear notion of order of update operations (stores) to a location. This is generally managed at the cache-line granularity (64 on the Intel Core-i7). To be clear, the functional model above speaks of store buffers. These are implemented by so-called Memory Ordering Buffers (MOBs), which internally do out-of-order actions. The flushing of buffers then corresponds to the point when non-local threads (on the same core or otherwise) can see those stores. Further buffering occurs between various levels of the cache. However, in all cases the cache maintains a coherent view, so that if the store is visible to at least one hardware thread other than the one executing the store, then it is visible to all of them.

x86-64 Cache Protocol. Both the Intel and AMD implementations of the $x 86$ cache protocol are variations of the classic MESI protocol [8]. Conceptually, there can be four states for each location in a cache (managed on a cache-line granularity): Modified (this cache holds an exclusive copy and memory has a stale copy), Exclusive (this cache holds an exclusive copy, the memory copy is also valid), Shared (this cache holds a copy, but other caches possibly hold copies as well), and Invalid (this cache does not hold a current copy for this location). While the cache behaviour does not directly impact the functional correctness proof, it does have implications for the cost models we develop.

\section{Correctness and Progress Properties}

This section considers the functional correctness of the spin-lock, queue and pipeline/task farm implementations described above, working from first principles in terms of the basic $x 86-64$ memory operations and $x 86$-TSO consistency model. We first consider a key ordering relation, coherence order, then sketch soundness proofs for the spin-lock and queue implementations, and finally build on these to sketch soundness proofs for the skeleton implementations.

\subsection{Coherence Order}

A derived relation called coherence order naturally emerges from the x86-TSO memory consistency model described above. Coherence order is a total, linear ordering of Writes to a given memory location, organised in the order that the Writes affect the memory location (are flushed from their local buffers). 
Definition 7. Coherence Order. Given a set of write buffers, $W B_{i}$ containing tuples $\langle t, m, v\rangle$, where $t$ is the time that the Write is flushed from $W B_{i}, m$ is the memory location, and $v$ is the new value to be written to that location, then the coherence order for some memory location $m$ is defined to be the sequence

$$
\begin{gathered}
C O(m) \text { linear order over }\left\{\left\langle t_{j}, m, v_{j}\right\rangle \mid\left\langle t_{j}, m, v_{j}\right\rangle \in \bigcup_{i=1}^{n} W B_{i}\right\} \\
\text { s.t. } \quad \forall j, k .\left\langle t_{j}, m_{j}, v_{j}\right\rangle<_{C O(m)}\left\langle t_{k}, m_{k}, v_{k}\right\rangle \Longrightarrow t_{k}>t_{j} .
\end{gathered}
$$

Note that under this definition, it is not possible for two Writes to the same memory location to occur at the same time. Writes to different memory locations may, however, occur at the same time. This is consistent with the restrictions of physical memory. It is easy to show from this definition that no Thread can read values out of coherence order.

Lemma 1. Read Coherence Order. No Thread can read values out of coherence order.

Proof Sketch: Suppose that two Reads $r_{1}$ and $r_{2}$ in the same Thread, T, from the same memory location, $m$, occur in order, but that they return different values. Let us call the corresponding Writes $w_{1}$ and $w_{2}\left(w_{1} \neq w_{2}\right)$. We do a case analysis depending on whether $w_{2}$ is read by $r_{2}$ from the local write buffer or from memory. Suppose that $w_{2}$ is in the write buffer. Then $w_{2}$ cannot have been the last write in the write buffer at the time of the read $r_{1}$, and thus $w_{2}$ must be flushed at some later time, and definitely later than $w_{1}$. Suppose that, instead, $w_{2}$ is taken from memory. Then the local write buffer must be empty for that location. Now, either $r_{1}$ read $w_{1}$ from the write buffer, and therefore $w_{1}$ must have been flushed (before $w_{2}$ ), or it read it from memory, and again $w_{2}$ is in Coherence order before $w_{1}$.

\subsection{Functional Correctness of the Spin-Lock Implementation}

The spin-lock implementation needs to enforce two key properties: i) that at most one thread at a time possesses the lock; and ii) that all Writes that are made while the lock is held are always visible to any subsequent thread that acquires the lock. We show this by using the memory properties defined in the previous section, together with the code for the spin-lock implementation of lock and unlock that was given in Figure 5.

Lock acquisition and release. We assume that the only Reads/Writes to lockcell are made by the lock and unlock functions. We also assume that unlock is called only when safe, i.e. when the calling Thread possesses the lock, and that lock is called only when the calling Thread does not possess the lock. It follows that the only values that lockcell can contain are 0 (the unlocked value) and 1 (the locked value). 
Theorem 1. Lock acquisition and release. Under the assumptions of the previous paragraph,

- following a call to lock, the calling Thread will possess the lock, and no other Thread will possess the lock; and

- following a call to unlock, the calling Thread will no longer possess the lock until it has successfully called lock again.

Proof Sketch: Both of the above parts can be proved simultaneously by induction on the lock and unlock calls in an execution trace, and by case analysis of the write buffer state at every lock. The key operation is the lock function, which is called when acquiring the lock. We return from this function exactly when the internal loop exits, that is when old_value is not 1 . This means that old_value is 0 , or in other words, that lockcell held 0 in the final iteration of the loop and is now 1 . Since the only way to update lockcell with a locked (1) value is via an Exchange, this will always appear directly in memory, and never stay in the write buffer. In contrast, an unlocked (0) value can stay in the buffers, since this is done by a simple Write. Since only the Thread that has successfully acquired the lock ever calls the unlock function, and since it does this precisely once for each lock acquisition, there will only ever be at most one unlock value $(0)$ for lockcell in any of the write buffers. There are now two cases to consider.

Case 1: Consider first the case when no write buffer contains an unlock value. If lockcell holds 1 , then the lock is already held. No lock acquisition can succeed until lockcell becomes 0 . Conversely, if lockcell holds 0 , the lock is free. Any isolated lock acquisition will successfully acquire the lock, but because of the atomic nature of the Exchange, if there are multiple simultaneous acquisition attempts then only one of these can succeed. Furthermore, because of coherence on lockcell, no subset of threads can disagree on which lock acquisition succeeded.

Case 2: The second case occurs when some Thread T's write buffer contains an unlock value. It follows that lockcell must still be locked, and therefore no Thread can acquire the lock. However, thread $T$ has released the lock. Now either the unlock value must eventually flush by itself, and we will then be in Case 1 above, or $T$ can attempt to re-acquire the lock, in which case the first action of the Exchange will be to flush the write buffer.

We thus obtain the first key property of the lock implementation, that the lock, from an initially unlocked position, flips between the locked and unlocked states, and because of coherence, no set of threads can disagree about which thread was responsible for each lock and unlock operation in that sequence. In other words, two distinct threads never think that they hold the lock at the same time. 
Synchronisation. We now turn to the second key property, that the lock acquisition and release operations collectively provide synchronisation. More precisely, following the acquisition of a lock, a thread should have an identical view of the shared memory to that of the unlocking thread at the immediately preceding lock release. This is not an immediate result, since the two threads may be different, and the view of the shared memory is mediated by the write buffers of the cores that are involved.

Theorem 2. Synchronisation. Assuming shared-memory accesses are only performed by threads holding a lock, all Writes made by a Thread between a successful call to lock and the following call to unlock are visible to the next Thread that acquires the lock.

Proof Sketch: We observe that each write buffer is emptied in a FIFO manner, and that each Thread has its own write buffer. Writes to shared memory locations within the critical section controlled by the lock are initially buffered in the write buffer for the Thread that acquired the lock. The lock release is itself a Write, that is placed in the buffer after all these Writes. We proved above that a new lock is only acquired when the unlocking Write is flushed from the buffer. Since the write buffer is FIFO, this implies that all the preceding Writes in the critical section must have already been flushed to the Memory. Now turning to the lock acquisition, since a lock acquisition involves an Exchange, which completely flushes a Thread's write buffer, any Read to the shared locations (in case of multiple accesses to one location, the first such) by the acquiring Thread must read its value from memory. It follows that synchronisation is obtained.

\subsection{Progress of the Spin-Lock Implementation}

When acquiring a lock, the Thread calling the lock function will loop until it succeeds. Conversely, the unlock function will always succeed. Progress of the system depends on two assumptions about the memory system. Firstly, if there are multiple contending Exchanges, exactly one will succeed. Secondly, all write buffers will eventually flush their contents to Memory. The first assumption is required to allow progress in the case of contending lock-acquires when the lock is free. The second assumption is required to allow progress when one thread has released a lock and other threads are waiting to acquire the lock. Note that if the same thread later wants to acquire the lock, the Exchange within lock will automatically flush the buffers. This is the progress condition for hardware exchanges that was discussed above. 


\subsection{Functional Correctness of the Queue Implementation}

Recall that the pseudo-code for the queue implementation was shown in Figure 4. We will assume that the queue is only touched by the qget and qput functions that are defined here, and the underlying lock location is not directly accessed by other code. We first verify that the queue implementation validates the assumptions made in the proof of the spin-lock implementation. First, since the only code that affects lockcell are the calls to the lock and unlock functions, it is easy to see no other code touches lockcell. Furthermore, on every control flow path, the unlock function is only called after having acquired the lock, it is not called more than once, and it is definitely called before either qget or qput returns. Moreover, any access to the queue and its fields occurs in program-order between a lock and an unlock from the same thread. Now we argue that the queue properties are ensured by the operations. There are three key properties:

1. The qget operation returns with a value that was previously placed in the queue.

2. A value that is placed once in the queue is never removed twice.

3. FIFO order is maintained for the queue.

For 1), the loop in the qget function can only be exited by the break statement. This means that when the loop exits, we know that the queue is non-empty, and furthermore, that the Thread that called qget holds the lock. Thus no other thread can remove elements from the queue, and it follows that the front operation will be able to find and remove at least one value. For 2), since the front operation completes before the lock is released, a value cannot be removed more than once. For 3), since only one thread can execute either qget or qput at a time, the queue follows sequential semantics. Therefore values are removed in the order that they were put into the queue.

\subsection{Progress of the Queue Implementation}

Since there is only one lock per queue, there is no possibility of deadlock. Progress depends on the progress properties of the underlying spin-lock implementation, in that if there are multiple contending qget and qput calls, at least one must succeed. Progress also depend on the fairness of the spin-lock implementation, which is an additional assumption. To see why, consider the case when the queue is empty, and there are one or more qget operations contending with a qput operation. An unfair implementation could allow a qget operation to succeed in acquiring the lock, notice that the queue is empty, release the lock, and immediately acquire the lock again without allowing the qput operation to acquire the lock. In this case, the qput would be starved, and the system as a whole would be prevented from making progress. 


\subsection{Functional Correctness of the Farm/Pipeline Skeletons}

As defined here, the pipeline and farm skeletons are both streaming operations, with workers applying functional operations to inputs to produce some output. The requirement is that all inputs that are placed in the input streams are processed to produce a corresponding output. Moreover, each input in a stream is processed precisely once. Each worker is associated with an input queue $Q_{i}=x_{1}, x_{2}, \ldots, x_{n}$ and an output queue $Q_{o}$, containing tasks and results, respectively. Each queue is guarded by its own lock, and is shared with one or more other workers. A worker obtains an input task from its input queue, applies its functional operation, and then places the result of the task on the output queue. It is easy to see that the assumptions that we need to ensure the correctness of the queue implementation (the queue is touched only by the qget and qput methods) are valid for both of the queues. Furthermore, any communication between workers occurs only through these queues.

Let us consider a parallel pipeline $f \mid g$. From the queue properties, by induction on the initial sequence in the input queue $x_{1}, x_{2}, \ldots, x_{n}$, and assuming that $f$ is finite, we can see that worker $f$ will produce results in the sequence $f\left(x_{1}\right), f\left(x_{2}\right), \ldots, f\left(x_{n}\right)$, and that all inputs will produce a corresponding output. Again, by the queue properties and by induction on this sequence, the next stage will produce the sequence $g\left(f\left(x_{1}\right)\right), g\left(f\left(x_{2}\right)\right), \ldots, g\left(f\left(x_{n}\right)\right)$. That is, $\forall j, 0<j \leq n . Q_{o}[j]=g\left(f Q_{i}[j]\right)$, and so the output queues $Q_{o}$ represents a map of $g . f$ over the elements in the input queues $Q_{i}$.

Now let us consider a task farm $\Phi(f)$. Suppose that the initial sequence in the input queue is $x_{1}, x_{2}, \ldots, x_{n}$. By the queue properties, each element is removed exactly once, and assuming that $f$ is finite, all workers will complete and put results $f\left(x_{i}\right)$ on the output queue. However, without further limitations, the order of the results in the output queue will now depend on the scheduling of each of the worker threads. Unlike the strong ordering for the pipeline, here we can only say that the output queue will contain $f\left(x_{1}\right), f\left(x_{2}\right), \ldots, f\left(x_{n}\right)$ in some permutation. It is not possible to make a strong statement about result ordering without further knowledge of the thread scheduler.

\subsection{Progress of the Farm and Pipeline Skeletons}

Since both the farm and the pipeline skeletons do not contain any high-level cycles, they satisfy progress. The only possible violations of progress are then when the queue operations are called, with either blocking or deadlocks. We have proved that the queue operations satisfy progress above (assuming a fair spinlock implementation), and thus proving progress under the same assumption here is almost trivial. The ease of this argument emphasises the advantages of taking a structured view. 


\section{Timing Models for $x 86-64$ Multicores}

Our overall objective is to obtain good timing predictions for parallel code running on $x 86-64$ multicores, based on a rigorous understanding of the underlying relaxed memory model. As mentioned in the hardware correspondence section, we consider a non-preemptive model. This means that our timing models directly apply when parallelism is less than or equal to the number of cores. Alternatively, if parallelism is greater than the number of cores, stronger fairness assumptions on the scheduler will have to be made to extend our timing models. In order to construct our timing cost models, we will use traces to describe the operation of each thread in the system, and then abstract over these to determine the overall timing behaviour of the system. A trace describes an actual execution of a thread in terms of the observable primitive operations that it performs. Since we are not interested in the values that our system produces, but only in the time that it takes to execute, we abstract over the actual computations that a thread performs between each memory access, using the abstract Compute operation to capture the time taken by actual computations. This paper considers only the costs associated with memory accesses. In the actual hardware, memory access costs depend on a variety of factors such as the presence or absence of a location in cache, the interconnect topology, the relative speeds of the various cache levels of the cache, etc. There are, thus, varying levels of realism that can be included in the cost model. We begin with a relatively naïve model, which just considers buffer sizes. As we will see, even a simple model like this already captures most of the important timing effects that we are interested in.

\subsection{Simple Average Timing Model}

Our first timing model estimates execution costs by assigning an average time to each kind of access. Our parameters are:

$T_{\text {Read }}$ the (average) time to read a location

$T_{\text {Write }}$ the (average) time to write a location

$T_{\text {Exchange }}$ the (average) time to exchange a location

We build up our model in stages, starting with the spin-lock implementation from Figure 5, then considering the queue implementation from Figure 4, and finally extending our model to the high-level farm and pipeline skeletons.

Spin-lock Timings. The cost of an unlock operation is just $T_{\text {Write }}$, while that of

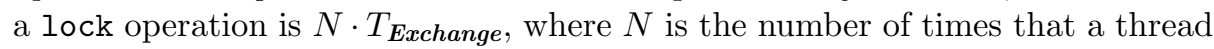
spins before acquiring the lock. Since each lock can only be held by one thread at a time, if a thread attempts to acquire a lock while it is held by another thread, it will spin uselessly. Once the lock is released, if $t$ threads are all trying to acquire the lock, only one will succeed. Assuming that the hardware allows precisely one thread to exchange successfully, it follows that it will take $t \cdot T_{\text {Exchange }}$ time before the lock is acquired by some thread. 
Queue Timings. Suppose that there are $n$ threads accessing the queue. In the worst case, they will all contend with each other. By the argument above, a qput operation therefore succeeds in time: $T_{\mathrm{qput}}=n \cdot T_{\text {Exchange }}+T_{\text {Write }}+T_{\text {Write }}$, i.e., $n \cdot T_{\text {Exchange }}$ for the lock; one $T_{W r i t e}$ when adding to the queue; and another $T_{W r i t e}$ for the unlock. Similarly, provided that the queue is not empty, a qget operation succeeds in time $T_{\text {qget }}=n \cdot T_{\text {Exchange }}+T_{\text {Read }}+2 T_{\text {Write }}$ i.e., $n \cdot T_{\text {Exchange }}$ for the lock; one $T_{\text {Read }}$ when reading the head of the queue; and two $T_{\text {Write }}$, one for writing the queue head and one for the unlock.

Farm and Pipeline Skeleton Timings. For the farm skeleton, suppose there are $n$ worker threads. The work done by each thread is a qget followed by the actual computation and finally a qput operation. Each of the queue operations has contention $n+1$. Thus, each thread takes time $T_{\text {qget }}+T_{f}+T_{\text {qput }}$ which simplifies to $2 \cdot(n+1) \cdot T_{\text {Exchange }}+4 \cdot T_{\text {Write }}+T_{\text {Read }}+T_{f}$. For the two-stage pipeline skeleton, there are two cases depending on which of the two stages dominates the time taken (the other being idle). If the first stage dominates (qualitatively, the second stage has insufficient work), then the first queue has contention $|f|+1$, the middle queue has contention $|f|+1$, and the second queue has contention 1 . The first stage thus takes time $T_{\text {qget }}+T_{f}+T_{\text {qput }}$ which simplifies to $2 \cdot(|f|+1) \cdot T_{\text {Exchange }}+5 \cdot T_{\text {Write }}+T_{f}$. while the second stage takes time $T_{g}$. The total time is the sum of the times for the two stages $2 \cdot(|f|+1) \cdot T_{\text {Exchange }}+$ $5 \cdot T_{\text {Write }}+T_{f}+T_{g}$. Conversely, if the second stage dominates (qualitatively, the second stage is saturated), then the first queue has contention $|f|+1$, the middle queue has contention $|f|+|g|+1$, and the second queue has contention 1 . The first stage takes time $T_{f}$, and the second takes $T_{\text {qget }}+T_{g}+T_{\text {qput }}$ which simplifies to $(2 \cdot|g|+|f|+1) \cdot T_{\text {Exchange }}+5 \cdot T_{\text {Write }}+T_{g}$ Again, the total time is the sum of the times for the two stages $T_{f}+(2 \cdot|g|+|f|+1) \cdot T_{\text {Exchange }}+5 \cdot T_{\text {Write }}+T_{g}$.

\subsection{Including Store Buffer Flushing}

A more realistic model would take into account that an exchange operation has two components: i) flushing the local store buffer, $T_{\boldsymbol{F} l}$; and ii) the actual exchange operation $T_{\boldsymbol{J} u s t \boldsymbol{X}}$. Naturally, the first component depends on the number of entries in the local buffer that are waiting to be flushed, $b$. It follows that $T_{\text {Exchange }}=b \cdot T_{\boldsymbol{F l}}+T_{\text {Just } \boldsymbol{X}}$.

Spin-lock Timings. Notice that the exchange operation is only used in the context of a tight loop in the lock operation. The first time, the store buffer must be flushed, but each successive time, the store buffer is already empty. The price of a flush is thus paid only once. Assuming that $t$ threads contend for a lock as before, and with the same assumption as before that a single exchange succeeds, then the cost of a lock operation is $b \cdot T_{\boldsymbol{F l}}+t \cdot T_{\boldsymbol{J} u s t \boldsymbol{X}}$. Here, $b$ is the (average) number of writes waiting in the buffers, which is between 0 and the size of the store buffer, $B$. The unlock operation has the same cost as in our first model.

Queue Timings. The qput operation now takes time: $b \cdot n \cdot T_{\boldsymbol{F l}}+n \cdot T_{\boldsymbol{J} u s t \boldsymbol{X}}+T_{\text {Write }}+$ $T_{\text {Write }}$, while the qget operation takes time: $b \cdot n \cdot T_{\boldsymbol{F l}}+n \cdot T_{\boldsymbol{J} \text { ust } \mathrm{X}}+T_{\boldsymbol{R e a d}}+2 \cdot T_{\text {Write }}$. 
Farm and Pipeline Skeleton Timings. A one-stage farm takes: $2 \cdot(n+1) \cdot b \cdot T_{F l}+$ $(n+1) \cdot T_{\text {Just } X}+4 T_{\text {Write }}+T_{\text {Read }}+T_{f}$. For a two-stage pipeline, the same analysis as before applies. If the first-stage dominates, the time is: $2 \cdot(|f|+1) \cdot b \cdot T_{F l}+$ $(2 \cdot|f|+|g|+1) \cdot T_{\text {Just } X}+5 \cdot T_{\text {Write }}+T_{f}$ and if the second-stage dominates, the time is: $(2 \cdot|g|+|f|+1) \cdot b \cdot T_{\boldsymbol{F l}}+(2 \cdot|f|+|g|+1) \cdot T_{\text {Just } X}+5 \cdot T_{\text {Write }}+T_{g}$.

\subsection{Predicted Speedups}

Speedup for Farms. We now use the cost model above to predict execution speedups for some example skeletons. First consider a simple one-stage farm with $n$ threads uniformly doing $T_{f}$ work. The computation takes time $n \cdot T_{f}$, which we can consider to be constant when calculating speedup. The total cost is as calculated for farms in $\S 5.2$. Assuming that $T_{f}$ is sufficiently large to dominate the time of a single Read, Write, or pure Exchange, the predicted speedup is approximately: $\operatorname{Speedup}_{\Phi} \simeq \frac{n \cdot W}{1+c \cdot n^{2}}$, where $W$ is a constant that depends on the total cost of computation, and $c$ is a constant that depends on the number of times that the lock is taken. Both these constants can be determined by instrumenting the code when it is run sequentially on just one core.

Speedup for Two-Stage Pipelines. Now consider a two-stage pipeline with $f$ firststage workers and $g$ second-stage workers, each uniformly doing work $T_{f}$ and $T_{g}$, respectively. Since the total work is constant, if the first stage is fully occupied then the predicted speedup will be: $\operatorname{Speedup}_{f \mid g} \simeq \frac{f \cdot W_{1}}{1+c_{1} \cdot f}(e q n .1)$, where $W_{1}$ is a constant that depends on the total cost of computation done in stage one (and can be approximated by the cost of a single thread in the first and a single thread in the second stages), and $c_{1}$ is a constant that depends on the number of times that the lock on the first queue is taken. If the second stage is saturated, then the predicted speedup will be: $\operatorname{Speedup}_{f \mid g} \simeq \frac{f \cdot W_{2}}{1+c_{2} \cdot(f+g)}$ (eqn. 2), where again $W_{2}$ is a constant depending on the total cost of computation, and $c_{2}$ is a constant depending on the number of times the lock on the second queue is taken. Constant $W_{2}$ will be $T_{g} / T_{f}$ times the constant $W_{1}$ above (and thus constant for a particular application). The stage that dominates depends precisely on which of the values from eqn. (1) or (2) is lower, i.e. precisely when the second stage becomes saturated. The final speedup is thus: $\operatorname{Speedup}_{f \mid g} \simeq \min \left(\frac{f \cdot W_{1}}{1+c_{1} \cdot f}, \frac{f \cdot W_{2}}{1+c_{2} \cdot(f+g)}\right)$. 


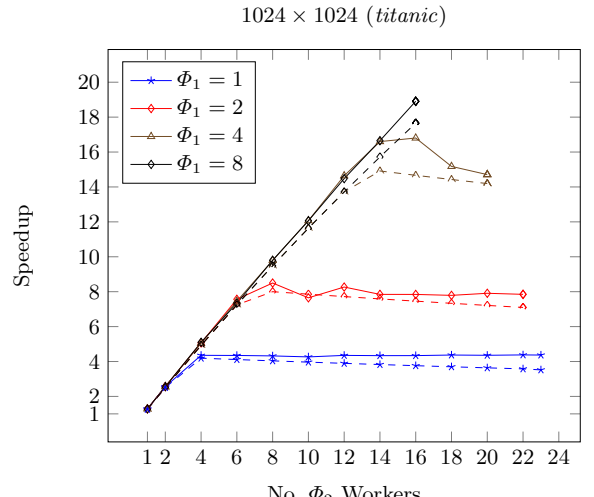

$2048 \times 2048$ (titanic)

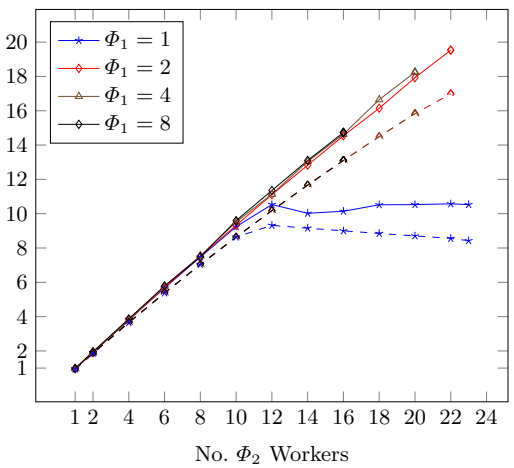

$2048 \times 2048$ (lovelace)

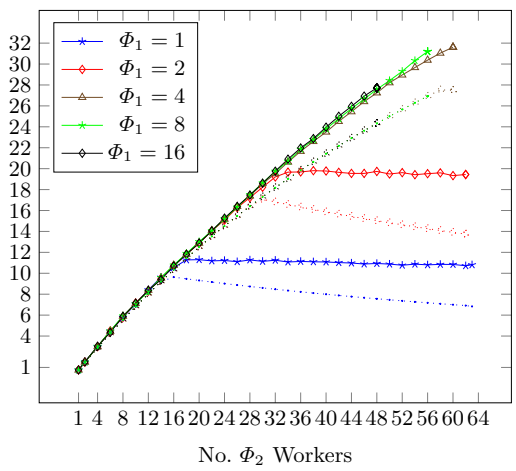

Fig. 6. Image Convolution $\left(\Phi_{1}(r) \mid \Phi_{2}(p)\right)$ on titanic and lovelace. Dashed/lighter lines are predictions. Note that the number of $\Phi_{1}+\Phi_{2}$ workers $\leq$ total hardware threads (24 for titanic/64 for lovelace).

\section{Experimental Validation}

We evaluate our cost models against real execution costs using a number of different benchmarks, running on three different x86-64 multicores. The main system that we use (titanic) is a $2.4 \mathrm{GHz} 24$-core, AMD Opteron 6176 architecture, running Centos Linux 2.6.18-274.e15, and gcc 4.4.6. We also use ladybank, a $2.93 \mathrm{GHz} 8$-core (plus hyperthreading) Intel Xeon X5570 architecture, running GNU/Linux 2.6.32-358.6.2.el6, and lovelace, a 2.3GHz 64-core, AMD Opteron 6376 architecture, running GNU/Linux 2.6.32-279.22.1.el6, which we used to test scalability for a limited set of experiments. All the results reported here are averages of 10 runs on an idle machine (but not in single-user mode). All speedups are absolute results against the original sequential versions. 


\subsection{Image Convolution}

Image convolution is widely used in image processing applications such as blurring, smoothing or edge detection. The convolution algorithm is a composition of two functions $r \circ p$, where $r$ reads in an image from a file and $p$ processes the image by applying a filter. This process is typically applied to a stream of input images, and produces a stream of output images. For each pixel in the input image, the filtering stage consists of computing the scalar product of the filter and the window surrounding the pixel:

$$
\text { output_pixel }(i, j)=\sum_{m} \sum_{n} \text { input_pixel }(i-n, j-m) \times \text { filter_weight }(n, m)
$$

For our benchmark tests, we parallelise the convolution using a two-stage pipeline, where each stage is a farm: $\Phi_{1}(r) \mid \Phi_{2}(p)$. Speedup results for two different image sizes on the 24-core titanic machine are shown on the left and centre of Figure 6 , with each worker in either farm allocated to its own core. Speedups are recorded against the sequential version of the algorithm. The solid lines show the actual execution speedups and the dashed lines show the speedup predictions. The $x$ axis is the number of $\Phi_{2}$ workers and each line corresponds to a fixed number of $\Phi_{1}$ workers. In all cases, the predicted speedups closely match the actual results, giving a correct lower bound prediction of the actual speedup. For the $1024 \times 1024$ images, the best speedup is 18.90 for $\Phi_{1}=8$ and $\Phi_{2}=16$ workers. Our cost model predicts a speedup of 17.65 (within $7 \%$ of the actual value). We observe three knees in the graph, where the speedups flatten out: for $\Phi_{1}=1$ and $\Phi_{2}=4$; or $\Phi_{1}=2$ and $\Phi_{2}=6$; and at $\Phi_{1}=8$ and $\Phi_{2}=14$. In all cases, our cost models correctly predict both the knee and the speedup. For the $2048 \times 2048$ images, the best speedup is 19.53 for $\Phi_{2}=22$ and $\Phi_{1}=2$, where our cost model predicts a speedup of 16.98 (within $14 \%$ of the actual value). We observe one knee, at $\Phi_{1}=1$ and $\Phi_{2}=12$, which our cost model also correctly predicts. The right-hand graph in Figure 6 investigates the scalability of our approach, showing speedup results for $2048 \times 2048$ images on the 64 -core lovelace machine. The best speedup that we obtain, at $\Phi_{1}=4$ and $\Phi_{2}=60$, is 31.6, versus a predicted speedup of 27.48 (that is, within $14 \%$ of the actual value). Speedup predictions for the remaining number of $\Phi_{1}$ workers (4,8 and 10) are consistent with the actual speedups, showing that the cost model correctly predicts identical speedup in all three cases.

\subsection{Cholesky Decomposition}

Cholesky Decomposition is used in linear algebra, comprising the decomposition of a Hermitian, positive-definite matrix into the product of a lower triangular matrix and its conjugate transpose. Our implementation uses a task farm, $\Phi_{1}$, to model the decomposition stage. Speedup results for a $1024 \times 1024$ matrix are shown in Figure 7. The top set of results shows speedups for the 24-core titanic machine (an AMD architecture) and the bottom set of results shows the corresponding speedups on the 8-core (hyperthreaded) ladybank machine (an 

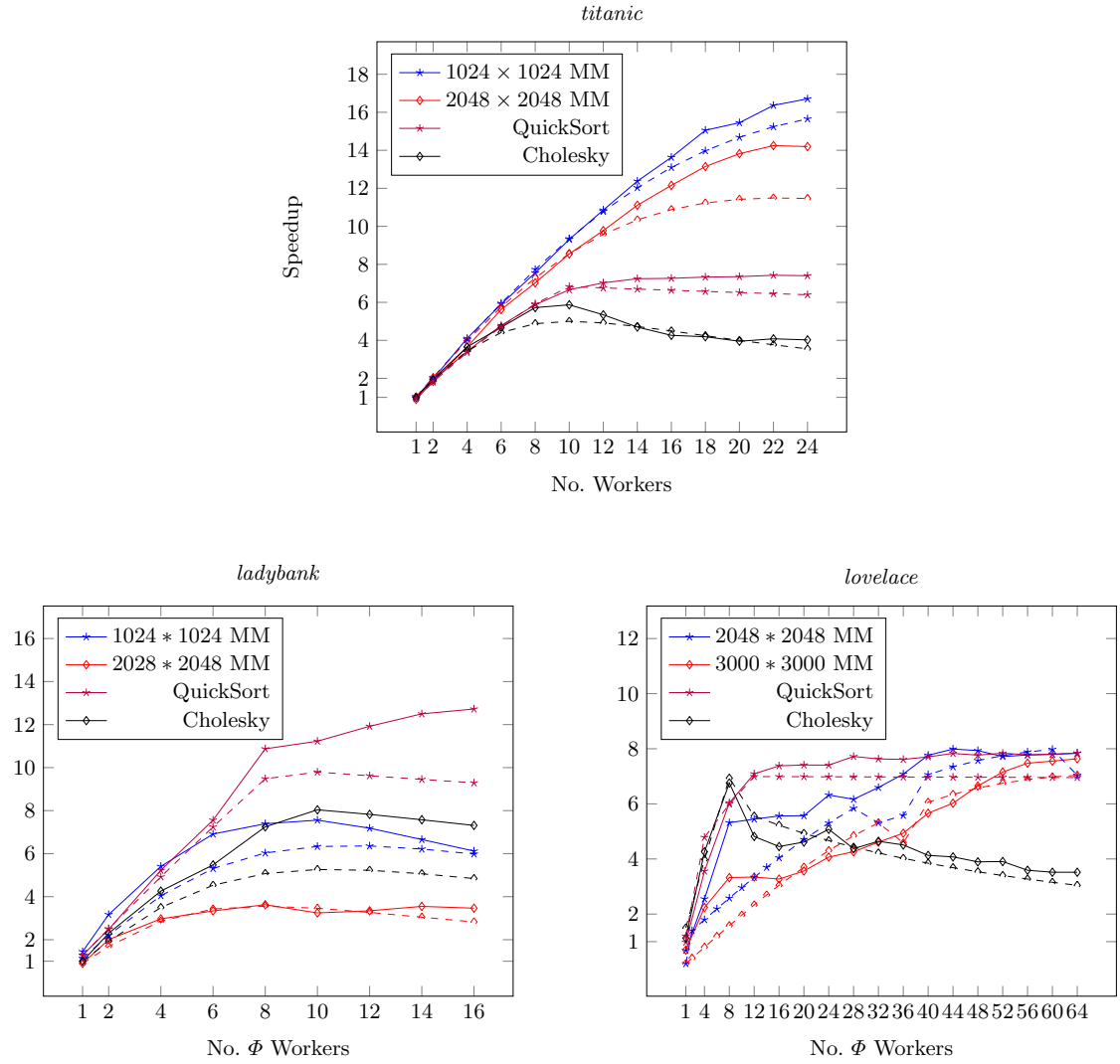

Fig. 7. Speedups for Cholesky Decomposition, Matrix Multiplication and QuickSort, using single task farms, on titanic, ladybank and lovelace. Dashed lines are predictions.

Intel Xeon architecture). For titanic, the best actual speedup is 5.86 for 10 workers, versus a best predicted speedup of 5.0 (also for 10 workers). The cost model also gives an almost perfect prediction, giving a lower bound between 6-12 workers. For ladybank, the best actual speedup is 8.03 for 10 workers, versus a predicted speedup of 5.26 for 10 workers. This is less accurate than for the AMD architecture, perhaps showing some additional complexities in the memory model that would repay further investigation. The cost model does, however, correctly predict a lower bound on the speedup in all cases. 


\subsection{Matrix Multiplication}

The product of two matrices, $A$ and $B$ is defined as:

$$
(A B)_{i, j}=\sum_{k=1}^{m} A_{i k} \cdot B_{k j}
$$

As for Cholesky decomposition, this can also be implemented using a task farm. with Figure 7 giving speedup results for $1024 \times 1024$ and $2048 \times 2048$ matrices on both titanic and ladybank. For ladybank, the $1024 \times 1024$ example gives a speedup of 7.55 on 10 cores, versus a prediction of 6.33 . Once again, the cost model correctly predicts lower bounds on speedup, predicting, for example, a speedup of 5.98 on 16 cores versus an actual speedup of 6.12 . For the $2048 \times 2048$ execution, the best speedup is 3.54 on 14 cores, versus a predicted speedup of 3.04. For titanic, the $1024 \times 1024$ example gives a best speedup of 16.7 on 24 cores versus a predicted speedup of 15.65. Likewise, for the $2048 \times 2048$ example, we obtain a best speedup of 14.19 versus a predicted speedup of 11.46 . In both cases, overall speedups are better for smaller matrices. This is due to lower communication costs. As with the Cholesky example, in all cases our cost models correctly predict a lower bound on speedup. In the best case, the prediction is within $3 \%$ of the actual speedup.

\subsection{QuickSort}

Figure 7 shows speedup results for a divide-and-conquer implementation of the classical QuickSort algorithm mapped to a task farm. All instances sort the same $10^{9}$ element list of randomly generated integers, with a threshold size of $10^{5}$. The best actual speedup on ladybank was 12.72 versus a predicted speedup of 9.29. For 8 and 10 workers, we observe a super-linear speedup of 10.87 for 8 workers and 11.2 speedup for 10 workers. While we do not have a precise explanation for this, our experiments showed that the effect is repeatable, and is presumably therefore some hardware effect. What is important is that our cost model correctly predicts a lower bound on speedup, showing a similar curve to the actual executions, with a knee at 8 workers. For titanic, speedup increases well from 1 to 10 workers, with an actual speedup of 6.6 on 10 workers versus a predicted speedup of 6.8 - one of the few cases where the cost model yields a (slight) over-estimate of the speedup. Beyond this point, speedup still improves, but only slightly, up to a maximum of 7.4 speedup on 24 cores (versus a prediction of 6.4). As with the other examples, the cost model closely predicts the actual speedup. 

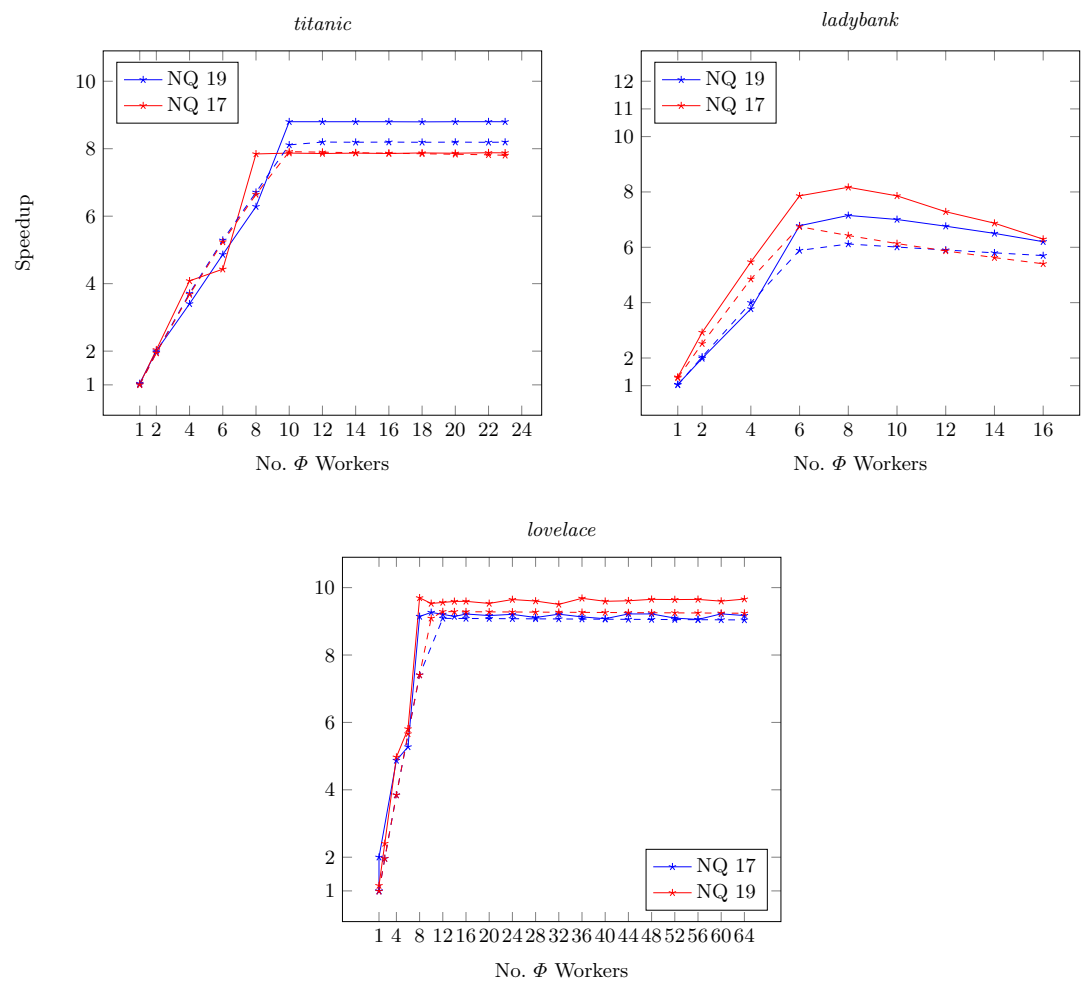

Fig. 8. Speedups for NQueens $(g \mid \Phi(s))$ on ladybank and titanic. Dashed lines are predictions.

\subsection{NQueens}

NQueens involves placing $n$ queens on an $n * n$ chessboard, so that no two queens may attack each other, according to the usual rules of chess. The solution requires that no two queens occupy the same row, column or diagonal. In our implementation, we have modelled the NQueens problem as a two-stage pipeline, $g \mid \Phi(s)$, where $g$ is the stage that generates all positions of the queens, and $s$ is the stage that solves the position. Speedup results for this implementation of NQueens are shown in Figure 8 for a $15 \times 15$ board, which produces 15 possible positions in the first stage, and for a $19 \times 19$ board, which produces 17 possible queens in the first stage. As with the other examples, the predicted speedups closely model the actual speedups. There is one over-prediction: for 6 workers on the $19 \times 19$ board, where we observe a discontinuity in the actual speedup. Otherwise, the predictions closely mimic the actual results that we obtain. The best speedups are 8.8 for 10 workers on a $19 \times 19$ board and 7.87 for 10 workers on a $17 \times 17$ board. 

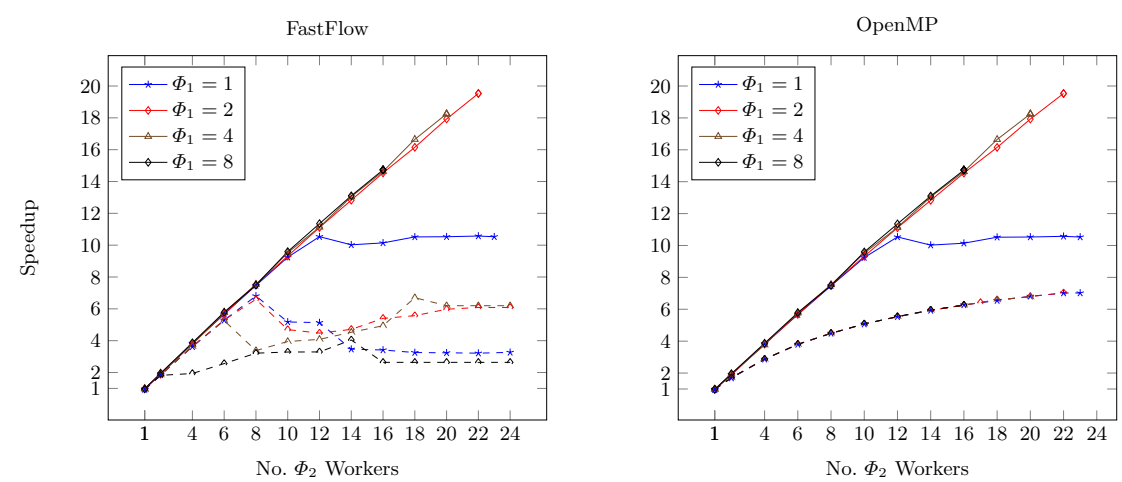

Fig. 9. Speedups for our implementation (solid lines) versus FastFlow (above, dashed lines), and OpenMP (below, dashed lines); Convolution of 500 images, size $2048 \times 2048$ (titanic). The baseline sequential performance is identical in each case.

\subsection{Comparison with other Techniques}

In order to demonstrate the efficiency of our queue and locking implementations, we have compared speedups for the Image Convolution example against those for two other state-of-the-art parallel implementations: OpenMP [9] and FastFlow [10]. Figure 9 compares all three implementations using $2048 \times 2048$ images on titanic. The top graph compares our implementation against FastFlow, and the bottom against OpenMP. In both cases, the speedup from our implementation is shown using solid lines, and the dashed lines show the speedups for FastFlow/OpenMP. All speedups were measured against the same sequential implementation (which took 768.75 seconds). In order to correctly compare the parallelism structures, we implemented the same two-stage pipeline in all three systems, using equivalent farm and pipeline structures in FastFlow, and two dynamic for-loops separated by a barrier synchronisation in OpenMP. The barrier is necessary to avoid the second stage processing images that have not yet been generated by the first stage, and is a natural translation of the parallelism structure that is used in the other implementations. As Figure 9 shows, the FastFlow implementation is comparable to ours up to 8 workers for $\Phi_{2}$ and for all versions of $\Phi_{1}$, but speedup reduces drastically after this point, whereas our implementation scales well up to 23 cores. The FastFlow implementation also carries further overhead in the form of two additional dedicated cores per task farm, which is not needed in our implementation. The OpenMP implementation scales better than FastFlow, but begins to flatten out at about $6 \Phi_{2}$ workers, where our implementation continues to scale well to 23 cores. 


\section{Related Work}

Timing issues are critically important for parallel and concurrent execution, and they have therefore been widely studied in the literature. However, despite their prevalence in real hardware, very little work considers relaxed memory models. This paper represents the first attempt of which we are aware to consider the precise impact of relaxed memory models on functional correctness, deadlock and timing. Correctness of the spin-lock protocol on x86-TSO has been previously proved using a semantic criterion (triangular-race freedom [11]) derived from the $x 86$-TSO model used here $[6,5]$. In contrast, our new proof proves functional correctness and deadlock freedom directly over the operational model of the actual $x 86-64$ instructions $[6,5]$. Our proof has been composed with a proof for queues and thence skeletons, and furthermore clearly isolates necessary hardware assumptions for progress. We also consider, for the first time, the crucial issue of execution time. Burckhardt et al [12] verify spin-locks on TSO by adapting a modified version of linearisability, and thus implicitly obtain compositionality. However, they ignore the progress and fairness constraints that we uncover, and also do not treat execution time.

Specific algorithmic skeletons are frequently associated with timing cost models [13]. However, these are obtained through measurement rather than being systematically derived from machine-level models, as here. Much of the work on developing cost models for parallel execution has focused on data parallelism. The Parallel Random-Access Machine (PRAM) execution model [14] acts as a theory of complexity for parallel algorithms on idealised shared-memory SIMD machines. In the basic PRAM execution model, basic computations and sharedmemory accesses are both assumed to take unit time. Unfortunately, PRAM costs underestimate actual machine execution costs, but in an unpredictable way [15]. The Bulk Synchronous Parallel (BSP) model [16] extends the PRAM model in a more realistic way, introducing a synchronising communication step after each set of computation steps. Lisper [17] has investigated the use of a Bulk Synchronous Parallel skeleton for determining worst-case execution times, but only informally, and not in the context of real processor models. Skillicorn and Cai have likewise developed a high-level cost calculus for data parallel computations [15], based on the shape of data structures, and known properties of primitive parallel operations, but have not based this on a strong machine-level semantics. Blelloch and Greiner have demonstrated provable time and space bounds for nested data parallel computations in NESL [18]. To date, none of these models have therefore been derived from first principles for real multicore architectures with relaxed-memory models. The model we give here thus represents a significant step in determining accurate cost models for algorithmic skeletons and other structured parallel forms on modern processor architectures. 
The $\mathrm{C} / \mathrm{C}++11 / \mathrm{C}++14$ standards provide atomic operations that support various kinds of weak memory models $[19,20]$. They could be used to implement the Fence and Exchange operations, but do not support higher-level parallel structures, such as the structured algorithmic skeletons that we have used here. Boehm and Adve [21] consider the foundations of this concurrency model. Adve and Boehm [22] give a useful survey of weak memory models as of 2010.

\section{Conclusions}

This paper has developed new proofs of functional correctness for $x 86-64$ multicores and used these to derive cost models for structured parallel programs. For the first time, we have direct operational proofs of functional correctness and deadlock freedom for standard locking and queuing algorithms under the relaxed memory model used by common $x 86-64$ multicores. We have used these to build accurate cost models for parallel programs that are structured using common algorithmic skeletons. Our predictions match very closely to actual results: in most cases predicting a lower-bound to within $7 \%$ accuracy.

\subsection{Limitations/Further Work}

There are a number of obvious limitations to the work described here that would repay further work. Firstly, the queues that we have used here are unbounded. Extending our work to consider bounded queues should not be technically difficult, but would add some complexity to the queue definitions and would also require us to consider how "back-pressure" [23] from the demand on one queue impacts the production of values that feed that queue. Secondly, although it is very commonly used in practice, the locking mechanism that we have used here carries some, possibly significant, cost: by definition, when a processor is executing a spin-lock, it is wasting energy and not doing any useful work. More efficient notification or "lock-free" ${ }^{2}$ techniques would obviate this at the cost of a significantly more complex proof and rather more complex cost model. We have therefore chosen not to do this here. Thirdly, and more seriously, as with most work on algorithmic skeletons, we have not considered any form of feedback, as in FastFlow's "farm/pipeline-with-feedback" skeletons [10]. Incorporating skeletons with feedback would allow the construction of more complex parallel programs, but would require us to determine fixed-points in the skeletons and to solve the resulting timing recurrence relations. Fourthly, we have ignored some hardware effects. In particular, while we have accounted for the behaviour of the cache and associated hardware when determining functional correctness and deadlock properties, we have not attempted to precisely model memory access behaviour in determining execution times, but have assumed that the sequential cost model properly accounts for such costs. We have also not modelled the processor instruction pipeline. Neither issue will affect functional correctness, but could, obviously, impact low-level timing accuracy.

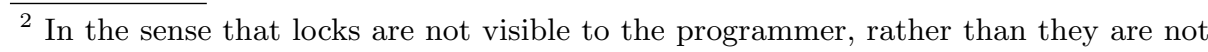
used by the hardware.
} 


\section{Acknowledgements}

This work has been partially supported by the EU Horizon 2020 grant "RePhrase: Refactoring Parallel Heterogeneous Resource-Aware Applications - a Software Engineering Approach" (ICT-644235), by COST Action IC1202 (TACLe), supported by COST (European Cooperation in Science and Technology), and by EPSRC grant EP/M027317/1 "C $\mathrm{C}^{3}$ : Scalable \& Verified Shared Memory via Consistency-directed Cache Coherence".

\section{References}

1. Cole, M.I.: Algorithmic skeletons: structured management of parallel computation. MIT Press, Cambridge, MA, USA (1991)

2. González-Vélez, H., Leyton, M.: A survey of algorithmic skeleton frameworks: highlevel structured parallel programming enablers. Software: Practice \& Experience 40(12) (2010) 1135-1160

3. Danelutto, M., Torquati, M.: A RISC Building Block Set for Structured Parallel Programming. In: Proc. 21st Euromicro International Conference on Parallel, Distributed, and Network-Based Processing (PDP '13). (2013) 46-50

4. Lamport, L.: How to Make a Multiprocessor Computer That Correctly Executes Multiprocess Programs. IEEE Transactions on Computers C-28(9) (September 1979) 690-691

5. Sewell, P., Sarkar, S., Owens, S., Zappa Nardelli, F., Myreen, M.O.: x86-TSO: A Rigorous and Usable Programmer's Model for x86 Multiprocessors. CACM 53(7) (July 2010) 89-97

6. Owens, S., Sarkar, S., Sewell, P.: A Better x86 Memory Model: x86-TSO. In: Proc. TPHOLs '09: Intl. Conf. on Theorem Proving in Higher-Order Logics, Springer LNCS 5674 (August 2009) 391-407

7. Intel: Intel 64 and IA-32 Architectures Software Developer's Manual Volume 3A: System Programming Guide, Part 1, §8.2.2. Intel (2013)

8. Papamarcos, M.S., Patel, J.H.: A Low-overhead Coherence Solution for Multiprocessors with Private Cache Memories. In: Proc. ISCA '84: 11th Annual International Symposium on Computer Architecture, ACM (1984) 348-354

9. Chapman, B., Jost, G., Pas, R.v.d.: Using OpenMP: Portable Shared Memory Parallel Programming (Scientific and Engineering Computation). The MIT Press (2007)

10. Aldinucci, M., Danelutto, M., Kilpatrick, P., Torquati, M.: Fastflow: High-level and Efficient Streaming on Multi-core. In: Programming Multi-core and Manycore Computing Systems, ser. Parallel and Distributed Computing. (2012)

11. Owens, S.: Reasoning about the Implementation of Concurrency Abstractions on x86-TSO. In: Proc. ECOOP '10: European Conference on Object-Oriented Programming, Springer (June 2010) 478-503

12. Burckhardt, S., Gotsman, A., Musuvathi, M., Yang, H.: Concurrent Library Correctness on the TSO Memory Model. In: Proc. ESOP '12: 21st European Conference on Programming Languages and Systems. (2012) 87-107

13. Hamdan, M.M.: A Survey of Cost Models for Algorithmic Skeletons. Technical report, Heriot-Watt University (1999)

14. Fortune, S., Wyllie, J.: Parallelism in Random Access Machines. In: Proc. STOC '78: 10th Annual ACM Symposium on Theory of computing, ACM (1978) 114-118 
15. Skillicorn, D.B.: A Cost Calculus for Parallel Functional Programming. Journal of Parallel and Distributed Computing 28 (1995)

16. Valiant, L.G.: A Bridging Model for Parallel Computation. Communications of the ACM (CACM) 33(8) (August 1990) 103-111

17. Lisper, B.: Towards Parallel Programming Models for Predictability. In: Proc. WCET '12: 12th International Workshop on Worst-Case Execution Time Analysis. Volume 23 of OpenAccess Series in Informatics (OASIcs). (2012) 48-58

18. Blelloch, G.E., Greiner, J.: A Provable Time and Space Efficient Implementation of NESL. In: Proc. ICFP '96: ACM SIGPLAN International Conference on Functional Programming. (1996) 213-225

19. Becker, P., ed.: Programming Languages - C++. ISO/IEC (2011)

20. Williams, A.: C ++ Concurrency in Action: Practical Multithreading. Manning Publications (2012)

21. Boehm, H.J., Adve, S.V.: Foundations of the C++ concurrency memory model. In: Proc. PLDI '08: 29th ACM SIGPLAN Conference on Programming Language Design and Implementation. (2008) 68-78

22. Adve, S.V., Boehm, H.J.: Memory Models: A Case for Rethinking Parallel Languages and Hardware. Communications of the ACM (CACM) 53(8) (2010) 90-101

23. Collins, R.L., Carloni, L.P.: Flexible filters: load balancing through backpressure for stream programs. In: Proc. EMSOFT '09: ACM SIGBED International Conference on Embedded Software. (2009) 205-214

\section{Dataset}

Data associated with this paper may be retrieved from http://dx.doi.org/ $10.5281 /$ zenodo. 58198 . 